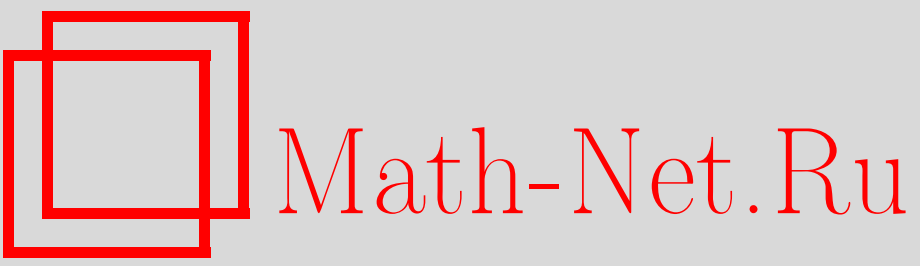

О. Н. Карпенков, Классификация трехмерных многоэтажных вполне пустых выпуклых отмеченных пирамид, УМН, 2005, том 60, выпуск 1, 169-170

DOI: https://doi.org/10.4213/rm1397

Использование Общероссийского математического портала Math-Net.Ru подразумевает, что вы прочитали и согласны с пользовательским соглашением

http://www.mathnet.ru/rus/agreement

Параметры загрузки:

IP: 3.85 .7 .115

26 апреля 2023 г., 12:33:13 


\title{
КЛАССИФИКАЦИЯ ТРЕХМЕРНЫХ МНОГОЭТАЖНЫХ ВПОЛНЕ ПУСТЫХ ВЫПУКЛЫХ ОТМЕЧЕННЫХ ПИРАМИД
}

\author{
О. Н. КАРПЕНКОВ
}

В этой заметке приводится результат решения одной из задач геометрии решетки целшіх точек и его приложение в теории многомерных цепных дробей. Автор выражает благодарность В.И. Арнольду за внимание к этой работе и полезные замечания.

Определения. Точка пространства $\mathbb{R}^{m}$ называется целой, если все ее координаты целые. Рассмотрим вьпукльй многоугольник $M$, все вершины которого - целые, и целую точку $A$ вне плоскости многоугольника $M$. Конус с вершиной $A$ и основанием $M$ назьвается целой выпуклой отмеченной пирамидой (с выделенной вершиной $A$ ). Целая выпуклая отмеченная пирамида называется вполне пустой, если она не содержит целшхх точек, отличных от вершины и целых точек основания. Два множества назьваются целочисленно-аффинно (целочисленно-линейно) әквивалентныцми, если существует аффинное (линейное) преобразование пространства $\mathbb{R}^{m}$, сохраняющее множество всех целых точек, которое переводит одно множество в другое. Плоскость назьвается целой, если она целочисленно-афффинно эквивалентна некоторой проходящей через начало координат плоскости, содержащей подрешетку решетки целых точек, ранг которой равен размерности плоскости.

Рассмотрим целую двумерную плоскость и целую точку в дополнении к этой плоскости. Пусть евклидово расстояние от данной точки до данной плоскости равно $l$. Обозначим через $l_{0}$ минималшное ненулевое евклидово расстояние до данной плоскости от целых точек трехмерной плоскости, натянутой на рассматриваемые двумерную плоскость и целую точку. Отношение $l / l_{0}$ называется иелочисленным расстоянием от данной целой точки до данной целой плоскости. Целая пирамида назьвается многоэтажнной, если целочисленное расстояние от вершины этой пирамиды до плоскости ее основания болшше единицы.

Определение многомерной цепной дроби по Клейну. Рассмотрим $n+1$ произвольные гиперплоскости в $\mathbb{R}^{n+1}$, пересечение которых состоит из одной точки - начала координат; пусть, кроме того, все выбранные гиперплоскости не содержат целых точек, отличных от начала координат. Дополнение к этим гиперплоскостям состоит из $2^{n+1}$ открытых ортантов. Рассмотрим один из них. Граница выпуклой оболочки множества всех целых точек, за исключением начала координат, входящих в замькание ортанта, называется парусом ортанта. Множество всех $2^{n+1}$ парусов назьвается $n$-мерной чепной дробъю, связанной с $n+1$ заданными гиперплоскостями. Пересечение гиперплоскости с парусом назьвается $k$-мерной гранью паруса, если оно содержится в некоторой $k$-мерной плоскости и гомеоморфно $k$-мерному диску. (См. [1] и [2].)

Формулировка основных утверждений. Через $\left(a_{1}, \ldots, a_{k}\right)$ в $\mathbb{R}^{m}$ при $k<m$ обозначим точку $\left(a_{1}, \ldots, a_{k}, 0, \ldots, 0\right)$. Оказывается, целочисленно-аффинных классов многоэтажных вполне пустых выпуклых трехмерных отмеченных пирамид немного.

ТеоРема 1. Любая многоэтажная вполне пустая выпуклая трехмерная отмеченная пирамида челочисленно-аффинно әквивалентна ровно одной из отмеченных пирамид списка " $M-W$ ".

Отметим, что до настоящего момента про компактные двумерные грани (парусов многомерных цепных дробей), плоскости которых расположены на целочисленном расстоянии, большем единицы, от начала координат, было известно следующее. Такие грани либо треуголшные, либо четырехугольные (см. работу Ж.-О. Муссафира [3]). Приведем полную целочисленно-линейную классификации таких граней.

СлЕдСтвиЕ 1. Любая двумерная компактная грань паруса многомерной чепной дроби, челочисленное расстояние от начала координат до плоскости которой больше единиць, является целочисленно-линейно эквивалентной ровно одной из граней с вершинами из следующего списка:

$$
\text { - }(2,-1,0),(2,-a-1,1),(2,-1,2),(2, b-1,1), \text { где } b \geqslant a \geqslant 1 \text {; }
$$

Работа выполнена при поддержке гранта НШ-1972.2003.1. 


\begin{tabular}{|c|c|c|c|c|}
\hline $\begin{array}{l}\text { Список } \\
\text { "М-W" }\end{array}$ & Параметры & $\begin{array}{c}\text { Коорд. } \\
\text { вершины }\end{array}$ & $\begin{array}{c}\text { Координаты } \\
\text { основания } \\
\end{array}$ & $\begin{array}{c}\text { Целочисленно-афффинный тип } \\
\text { основания }\end{array}$ \\
\hline$M_{a, b}$ & $b \geqslant a \geqslant 1$ & $(0,0,0)$ & $\begin{array}{l}(2,-1,0) \\
(2,-a-1,1) \\
(2,-1,2),(2, b-1,1)\end{array}$ & $(-a, 0) \underbrace{(0,1)}_{(0,-1)}(b, 0)$ \\
\hline$T_{a, r}^{\xi}$ & $\begin{array}{l}a \geqslant 1, r \geqslant 2, \\
0<\xi \leqslant r / 2, \\
\text { НОД }(\xi, r)=1\end{array}$ & $(0,0,0)$ & $\begin{array}{l}(\xi, r-1,-r), \\
(a+\xi, r-1,-r), \\
(\xi, r,-r)\end{array}$ & $(0,0) \quad(a, 0)$ \\
\hline$U_{b}$ & $b \geqslant 2$ & $(0,0,0)$ & $\begin{array}{l}(2,1, b-1) \\
(2,2,-1) \\
(2,0,-1)\end{array}$ & $(0,-1) \underset{1}{(0,1)}(b, 0)$ \\
\hline$V$ & & $(0,0,0)$ & $\begin{array}{l}(2,-2,1) \\
(2,-1,-1) \\
(2,1,2)\end{array}$ & $\begin{array}{c}(-1,0) \\
(0,-2) \\
(0,-+\infty\end{array}$ \\
\hline$W$ & & $(0,0,0)$ & $\begin{array}{l}(3,0,2) \\
(3,1,1) \\
(3,2,3)\end{array}$ & $(-1,-1)$ \\
\hline
\end{tabular}

- $(\xi, r-1,-r),(a+\xi, r-1,-r),(\xi, r,-r)$, әде $a \geqslant 1$, a $\xi$ и $r$ взаимно простыи, причем $r \geqslant 2 u 0<\xi \leqslant r / 2$

- $(2,1, b-1),(2,2,-1),(2,0,-1)$, әде $b \geqslant 2$;

- $(2,-2,1),(2,-1,-1),(2,1,2) u(3,0,2),(3,1,1),(3,2,3)$.

Все треугольные грани списка реализуются для парусов любой размерности, большей единицы. Все четырехугольные грани списка реализуются для парусов любой размерности, большей двойки, и не реализуются для парусов размерности два.

\section{СПИСОК ЛИТЕРАТУРЫ}

[1] В. И. Арнольд. Цепные дроби. М.: МЦНМО, 2002. [2] О. Н. Карпенков // Функц. анализ и его прил. 2004. Т. 38. № 2. С. 28-37. [3] J .-O. Moussafir. Voiles et Polyèdres de Klein: Géométrie, Algorithmes et Statistiques // Docteur en sciences thése. Paris: Université Paris IX Dauphine, 2000.

Московский государственньй университет им. М.В. Ломоносова E-mail: karpenk@mccme.ru
Представлено В. И. Арнольдом Принято редколлегией 23.11.2004 